\title{
EVALUACIÓN DE LA CALIDAD ECOLÓGICA DE QUEBRADAS ANDINAS EN LA CUENCA DEL RÍO MAGDALENA, COLOMBIA
}

\section{EVALUATION OF THE ECOLOGICAL QUALITY OF ANDEAN CREEKS IN THE MAGDALENA RIVER BASIN, COLOMBIA}

\author{
Esnedy Galeano-Rendón ${ }^{1}$, Lina María Monsalve-Cortes ${ }^{2}$, Néstor Javier Mancera-Rodríguez ${ }^{3 *}$
}

${ }^{1}$ Licenciada en Biología, Estudiante de Maestría en Ciencias. Universidad Nacional de Colombia, sede Medellín, e-mail: emgaleanor@unal.edu.co; ${ }^{2}$ Ingeniera Forestal, Especialista en Medio Ambiente y Geoinformática, e-mail: Immonsalc@unal. edu.co; ${ }^{3}$ Doctor en Biología. Profesor Titular. Universidad Nacional de Colombia, Sede Medellín, Departamento de Ciencias Forestales, Calle 59A No. 63-20, Bloque 20, oficina 211, Medellín, Colombia, e-mail: njmancer@unal.edu.co

Rev. U.D.C.A Act. \& Div. Cient. 20(2): 413-424, Julio-Diciembre, 2017

\section{RESUMEN}

Se evaluó la calidad ecológica de cuatro quebradas andinas afluentes de los ríos Nare y Guatapé, cuenca del río Magdalena, Colombia. Se realizaron cuatro muestreos entre junio de 2011 y febrero de 2012, en tres sitios por quebrada, para un total de 12 evaluados. Se recolectaron muestras de macroinvertebrados, utilizando el método D-net, con malla de $500 \mu \mathrm{m}$ y colección manual sobre sustratos. El hábitat del río y la vegetación ribereña fueron evaluados, mediante el establecimiento de estaciones de referencia y la aplicación del Índice de Hábitat Fluvial (IHF), el Índice de calidad de la vegetación de ribera Andina (QBR-And), adaptado para los ríos andinos, el Índice Biological Monitoring Working Party (BMWP/Col) y el índice ECOSTRIAND (ECOlogical STatus RIver ANDean). Se establecieron siete sitios de referencia, caracterizados por vegetación ribereña en ambos márgenes, sin alteraciones o con un bajo grado de alteración antrópica, los cuales, presentaron altos valores de IHF, QBR-And, $\mathrm{BMWP} / \mathrm{Col}$, indicando buena calidad ecológica para los ensamblajes de macroinvertebrados. Cinco sitios se determinaron como alterados con alta afectación antrópica, vegetación ribereña reducida o ausente en los dos márgenes, predominio de pastos y actividades agrícolas y bajos valores en los índices antes mencionados, lo que indica una menor calidad ecológica.

Palabras clave: Macroinvertebrados acuáticos, caracterización del hábitat, condiciones de referencia.

\section{SUMMARY}

The ecological quality of four Andean creeks tributaries of the Nare and Guatapé Rivers, Magdalena River Basin, Colombia was evaluated. Four field samplings were carried out between June 2011 and February 2012, at three sites for each creek, for a total of 12 sites evaluated. Macroinvertebrates samples were caught using the D-net method with mesh of $500 \mu \mathrm{m}$ and manual collection on substrates. The river habitat and riparian vegetation were evaluated through of the establishment of reference stations and the application of the River Habitat Index (IHF), the Riparian-Andean vegetation quality index (QBR-And) adapted for Andean rivers, the Biological Monitoring Working Party-Colombia index (BMWP / Col), and the ECOSTRIAND index (ECOlogical STatus Rlver ANDean). Seven reference sites were established, which were characterized by riparian vegetation in both sides, without alterations or with a low degree of anthropic disturbance, presented high values of IHF, QBR-And, BMWP/Col, indicating good ecological quality for macroinvertebrates and fish assemblage's. Five sites were determined as altered with high anthropogenic disturbance, riparian vegetation reduced or absent in the two margins, predominance of pastures and agricultural activities, and low values in the afore mentioned indexes, which indicates less ecological quality.

Key words: Aquatic macroinvertebrates, habitat characterization, reference conditions.

\section{INTRODUCCIÓN}

Los Andes tropicales forman parte de las regiones más propensas a experimentar altos niveles de pérdida de especies, dadas las actuales tendencias de deforestación, por la ex- 
pansión de la frontera agrícola y ganadera, la contaminación acuática, la introducción de especies, la construcción de hidroeléctricas, entre otros (Anderson \& Maldonado-Ocampo, 2011). En particular, las actividades agropecuarias afectan las características bióticas y abióticas de pequeñas quebradas de montaña en Colombia (Giraldo et al. 2014), con un fuerte impacto sobre la calidad del agua, causando graves reducciones de la diversidad biológica. Este es el caso de algunas quebradas que desembocan en los ríos Nare y Guatapé, en la cuenca del Río Magdalena, donde la deforestación se encuentra relacionada con la ampliación de pasturas para ganadería, para agricultura y el crecimiento de los asentamientos humanos. En consecuencia, las dinámicas de estos ecosistemas se ven influenciadas negativamente por la pérdida de cobertura arbórea de los márgenes y los efectos de compuestos químicos, como plaguicidas y fertilizantes, que llegan al cauce.

Para evaluar la afectación por diferentes actividades antrópicas y la calidad ecológica de los cuerpos hídricos, mediante el estudio de la diversidad de organismos presentes en ellos, es necesario contar con información de estaciones de muestreo, que sirvan de referencia (Acosta et al. 2009) y de métodos que permitan evaluar, de manera rápida y efectiva, la calidad ecológica de hábitats acuáticos y sus comunidades. Los sitios de referencia son escenarios con la menor interferencia humana posible y son útiles para medir el grado de alteración antrópica de otros sitios pertenecientes a la misma ecoregión (Karr, 1991; Hughes, 1995; Barbour et al. 2000; Bailey et al. 2004; Ligeiro et al. 2013; Pilièrea et al. 2014) y existen varias metodologías para definir las condiciones de referencia (Karr, 1981; Reynoldson et al. 1997; Stoddard et al. 2006; Whittier et al. 2007; Acosta et al. 2009; Moreno et al. 2009).

En este sentido, el estudio de calidad ecológica debe evaluar aspectos fisicoquímicos del agua, biológicos y físicos del hábitat. A nivel biológico, los macroinvertebrados acuáticos son indicadores más sensibles a la degradación del hábitat local, debido a su limitada movilidad (Kerans \& Karr, 1994) y sensibilidad a bajos niveles de contaminantes, que permite la detección rápida de la degradación del cuerpo hídrico (Jacobsen, 1998; Compin \& Céréghino, 2003). Por lo anterior, los ensamblajes de macroinvertebrados han sido usados como herramientas para determinar la integridad biótica de los ríos (Pilièrea et al. 2014; Piñón-Flores et al. 2014), permitiendo el desarrollo de más de cuarenta índices de biomonitorización (Herman \& Nejadhashemi, 2015).

Asimismo, es importante la evaluación de la calidad de las características físicas del cauce y de la ribera, debido a que los resultados obtenidos mediante estos índices físicos son fundamentales, al considerar que un buen estado del entorno natural incide en la calidad ecológica (Bonada et al.
2002). Lo anterior, se hace mediante el uso de índices, especialmente diseñados para ello, como el Índice de Hábitat Fluvial (IHF), que valora la capacidad del hábitat físico para albergar una fauna determinada (Pardo et al. 2002) y el índice de calidad de bosque de ribera (QBR), que considera que los bosques de ribera son indicadores de la gestión antrópica del territorio (actividades de agricultura, ganadería, construcción de vías y represas, entre otros) y son elementos clave para la calificación del estado ecológico de los ríos (SuárezAlonso et al. 2002).

Teniendo en cuenta, la importancia de la creciente transformación de los ecosistemas acuáticos y la pérdida de biodiversidad de los Andes colombianos por actividades antrópicas es fundamental integrar diferentes índices, que permitan evaluar la calidad ecológica y generar información necesaria para diseñar estrategias de conservación, de restauración y de protección de quebradas Andinas y bosques de ribera de estas zonas. El objetivo de este trabajo fue determinar la calidad ecológica de cuatro quebradas Andinas, con diferentes grados de perturbación antrópica, pertenecientes a la cuenca del río Magdalena, en el departamento de Antioquia, Colombia, mediante la utilización del Índice de Hábitat Fluvial (IHF), el Índice de calidad de la vegetación de ribera Andina (QBR-And), el índice Biological Monitoring Working Party (BMWP/Col), basado en el ensamblaje de macroinvertebrados acuáticos y el índice ECOlogical STatus RIver ANDean (ECOSTRIAND).

\section{MATERIALES Y MÉTODOS}

Localización y descripción del área de estudio: El trabajo, se realizó en las quebradas La Magdalena y Las Mercedes (afluentes del río Nare) y en las quebradas Peñoles y El Cardal (afluentes del rio Guatapé), ubicadas en los municipios de San Vicente, Concepción, San Rafael y San Carlos, Antioquia, Colombia (Tabla 1). En cada una de las quebradas, se seleccionaron tres sitios de muestreo, para un total de 12 estaciones y se describieron las características de uso de suelo, cobertura vegetal y grado de conservación. El territorio es montañoso, con topografía ondulada y las quebradas drenan hacia la cuenca media del río Magdalena, caracterizándose por ser torrenciales, de aguas muy claras y sin planicie aluvial (Mancera-Rodríguez et al. 2016). La zona presenta características de bosque húmedo tropical montano bajo, con una temperatura promedio de $18^{\circ} \mathrm{C}$, para San Vicente y Concepción y de $23^{\circ} \mathrm{C}$, para San Rafael y San Carlos y un patrón de precipitación bimodal con picos en mayo y octubre (Mancera-Rodríguez et al. 2016).

Caracterización del hábitat: Se valoraron los 24 atributos propuestos por Acosta et al. (2009), en el protocolo de evaluación de la calidad ecológica de ríos andinos (CERA), que incluyen la caracterización en cuatro niveles jerárquicos 


\begin{tabular}{|c|c|c|c|c|c|c|c|c|c|c|c|c|c|}
\hline 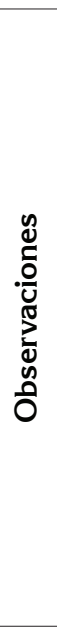 & & \multicolumn{3}{|c|}{ 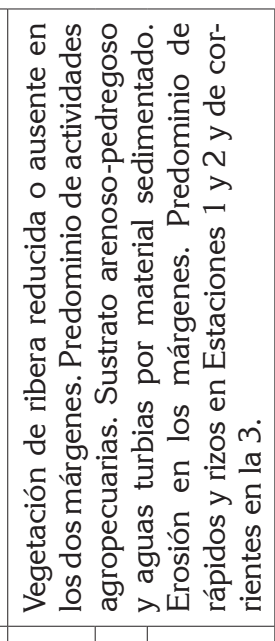 } & \multirow{2}{*}{ 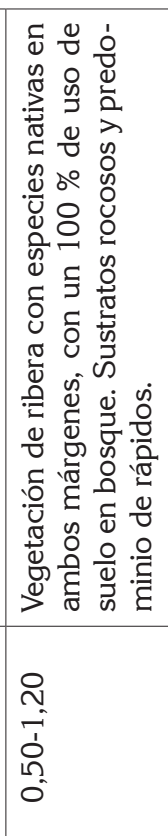 } & \multicolumn{2}{|c|}{ 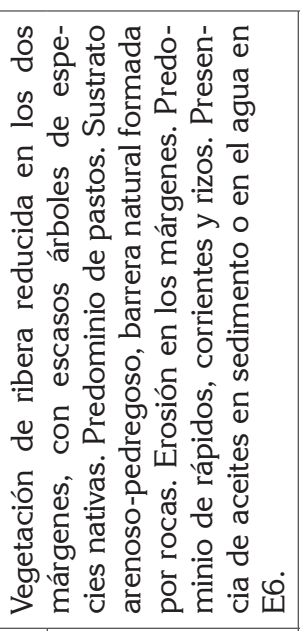 } & \multicolumn{3}{|c|}{ 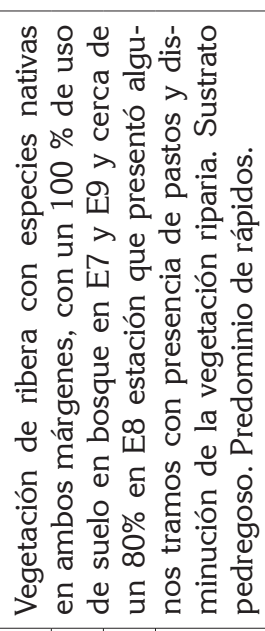 } & \multicolumn{3}{|c|}{ 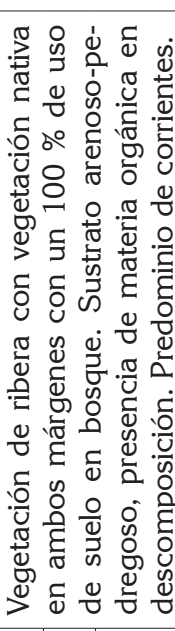 } \\
\hline 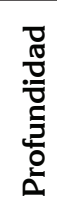 & & $\begin{array}{l}8 \\
0 \\
0 \\
0 \\
10 \\
0 \\
0\end{array}$ & $\mid \begin{array}{c}8 \\
8 \\
-1 \\
0 \\
+ \\
0 \\
0\end{array}$ & $\begin{array}{l}\stackrel{0}{N} \\
-\dot{0} \\
\dot{0} \\
\dot{0}\end{array}$ & & $\begin{array}{l}8 \\
8 \\
\dot{-} \\
\dot{o} \\
\dot{0}\end{array}$ & 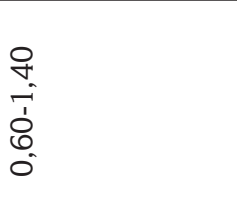 & $\begin{array}{c}0 \\
N \\
-1 \\
\\
0 \\
0\end{array}$ & $\mid \begin{array}{c}0 \\
+ \\
-1 \\
\vdots \\
0 \\
0 \\
0\end{array}$ & 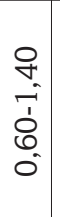 & & & 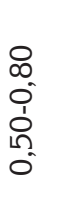 \\
\hline 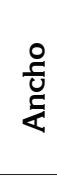 & 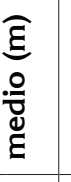 & $\stackrel{\sim}{N}$ & $\begin{array}{l}\dot{*} \\
\dot{\sigma}\end{array}$ & $\begin{array}{l}\stackrel{ \pm}{ \pm} \\
\end{array}$ & $\begin{array}{l}0 \\
\stackrel{0}{\circ}\end{array}$ & $\begin{array}{l}\stackrel{+}{n} \\
\stackrel{n}{n}\end{array}$ & $\stackrel{\llcorner}{M^{n}}$ & $\begin{array}{l}0 \\
\dot{\Xi}\end{array} \mid$ & $\begin{array}{l}\vec{m} \\
\vec{n}\end{array}$ & $\begin{array}{l}n \\
0 \\
0\end{array}$ & $=$ & & $\exists$ \\
\hline 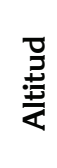 & 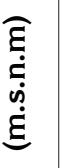 & $\underset{\stackrel{g}{N}}{\stackrel{O}{N}}$ & $\begin{array}{c}N \\
\infty \\
\infty \\
\sim\end{array}$ & $\begin{array}{l}\stackrel{1}{N} \\
\substack{\infty \\
\sim}\end{array}$ & $\underset{\sim}{\stackrel{\text { I }}{\simeq}}$ & $\mid \begin{array}{l}\mathscr{0} \\
\mathscr{0} \\
-1\end{array}$ & $\begin{array}{l}\stackrel{0}{\infty} \\
\stackrel{\cap}{2} \\
\sim\end{array}$ & $\begin{array}{l}\stackrel{\llcorner}{0} \\
\stackrel{\sim}{\simeq}\end{array}$ & $\begin{array}{l}0 \\
\stackrel{\sim}{\sim} \\
\end{array}$ & $\stackrel{\vec{D}}{\underset{v}{*}}$ & 寸 & $\frac{9}{3}$ & $\begin{array}{l}\infty \\
\infty\end{array}$ \\
\hline 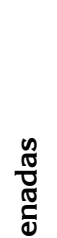 & 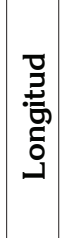 & 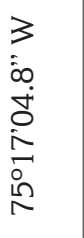 & 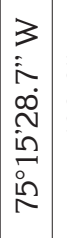 & 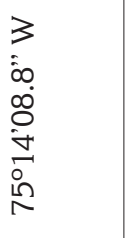 & $\begin{array}{l}3 \\
\vdots \\
\cdots \\
\infty \\
0 \\
0 \\
0 \\
0 \\
\stackrel{0}{0} \\
i\end{array}$ & 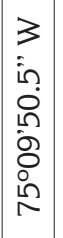 & 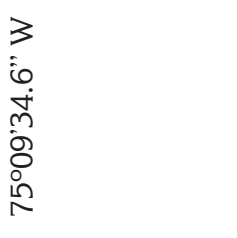 & 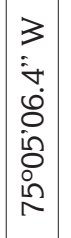 & 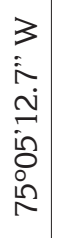 & 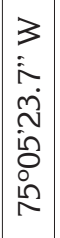 & $\begin{array}{l}3 \\
\text { v } \\
0 \\
0\end{array}$ & & 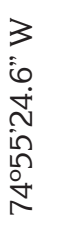 \\
\hline ¿ั & 胥 & $\begin{array}{l}z \\
i \hat{i} \\
i \\
\hat{j} \\
\sigma \\
\vdots \\
0\end{array}$ & $\begin{array}{c}z \\
\vdots \\
j \\
j \\
\sim \\
0 \\
0 \\
\vdots \\
0\end{array}$ & 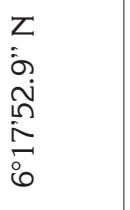 & 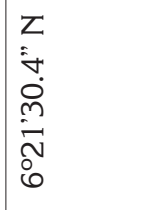 & 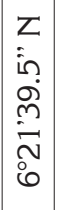 & 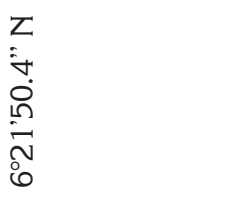 & \begin{tabular}{|l|}
$z$ \\
$\vdots$ \\
0 \\
$\dot{T}$ \\
$i n$ \\
$i$ \\
0 \\
0
\end{tabular} & $\begin{array}{l}z \\
\dot{j} \\
\dot{0} \\
0 \\
0 \\
0\end{array}$ & $\begin{array}{c}z \\
\vdots \\
\hat{N} \\
\hat{N} \\
0 \\
0 \\
0 \\
0\end{array}$ & 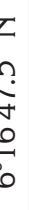 & & $\begin{array}{l}Z \\
0 \\
0 \\
0 \\
0 \\
0 \\
0 \\
0\end{array}$ \\
\hline 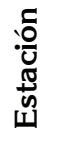 & & $\vec{\Psi}$ & $\widetilde{\Psi}$ & $\tilde{\uplus}$ & $\stackrel{\nabla}{\uplus}$ & 뇨 & 岀 & $\hat{\Psi}$ & $\mid \begin{array}{c}\infty \\
\ddot{山}\end{array}$ & ஐூ & $\vec{\nu}$ & $\vec{\nu}$ & $\stackrel{\sim}{\vec{w}}$ \\
\hline Uू & & $\frac{0}{\frac{\pi}{\pi}}$ & & & 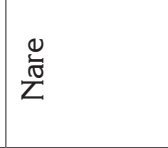 & & & $\mid \begin{array}{l}0 \\
0 \\
\frac{0}{0} \\
\frac{0}{0} \\
0\end{array}$ & & & & & \\
\hline $\begin{array}{l}\frac{\pi}{0} \\
\frac{\pi}{0} \\
\frac{0}{20} \\
0\end{array}$ & & 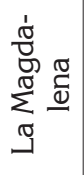 & & & 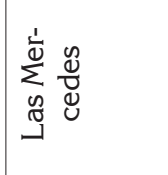 & & & 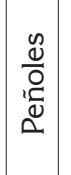 & & & & & \\
\hline
\end{tabular}


(cuenca, hidrología, tramo y lecho), para establecer las condiciones de referencia, a partir de las posibles presiones o impactos que en ellas se producen y que determinan la calidad ecológica de los ríos. Se consideraron como estaciones de referencia, aquellas con valores superiores a 100 puntos y que obtuvieron como mínimo 20 puntos en la valoración de cada nivel jerárquico, de acuerdo a la metodología propuesta por Acosta et al. (2009).

En una sección longitudinal de 100 m en cada estación, se describieron los tipos de micro hábitats específicos, como pozas, corrientes, rizos y rápidos, los impactos locales sobre la corriente, como barreras físicas, presencia de contaminantes o aceites y el uso del suelo predominante en las riberas, como agrícola, pecuario y de conservación de bosque. El ancho y la profundidad fueron medidos con flexómetro y una vara graduada en $\mathrm{cm}$. En cada una de las estaciones seleccionadas, se midieron las siguientes variables fisicoquímicas in situ: oxígeno disuelto (mg/L), saturación de oxígeno (\%) y temperatura superficial del agua $\left({ }^{\circ} \mathrm{C}\right)$, con medidor Hanna (HI9828/4-01) y pH, conductividad eléctrica $(\mu \mathrm{S} / \mathrm{cm})$ y sólidos totales disueltos (STD), con medidor Hanna (9811-5).

Obtención e identificación de macroinvertebrados acuáticos: Se realizaron cuatro muestreos, que incluyeron los períodos de transición de lluvia a seco (junio de 2011), transición de seco a lluvia (septiembre de 2011), lluvia (noviembre de 2011) y seco (febrero de 2012). Dentro de cada sección de muestreo, se seleccionaron 10 puntos al azar a lo largo del tramo de $100 \mathrm{~m}$ de la estación, según la metodología descrita por Roldán \& Ramírez (2008). En cada punto, los macroinvertebrados acuáticos se recolectaron manualmente sobre sustratos de vegetación, restos de madera sumergida, rocas y áreas de depositación, presentes en el cauce de las quebradas, con la ayuda de pinzas entomológicas. En las zonas con hojarasca y sedimento, la captura se hizo con una red tipo $D$ de $405 \mathrm{~cm}^{2}$ y poro de $500 \mu \mathrm{m}$; las muestras fueron llevadas al laboratorio de Ecología y Conservación de Fauna Silvestre de la Universidad Nacional de Colombia, sede Medellín. La identificación taxonómica, se realizó mediante el uso de las claves especializadas y las muestras se preservaron en alcohol al 70\% y están depositadas en el Museo Entomológico Francisco Luis Gallego de la Universidad Nacional de Colombia, sede Medellín (código MEFLG).

Evaluación de calidad ecológica: Se utilizaron los siguientes índices: a) el Î́ndice de Hábitat Fluvial (IHF), propuesto por Pardo et al. (2002), se calculó durante el periodo seco, cuando era menor el caudal y se apreció con mayor facilidad el sustrato y las características del canal, de acuerdo a lo sugerido por Munné et al. (2006) y b) el Índice de calidad de la vegetación de ribera Andina (QBR-And), adaptado por Acosta et al. (2009), a partir del Índice QBR (Munné et al. 2003), para riberas tipo 3 , que corresponden a una comunidad arbórea o arbustiva muy diversa. Las condiciones de vegetación en las riberas de todas las estaciones no tuvieron cambios durante los cuatro muestreos.

Para el análisis de la calidad biológica del agua, se utilizó el Índice BMWP-Col, de acuerdo a la escala adaptada para Colombia, por Roldán (2003) y algunos de los puntajes de tolerancia a la contaminación para los grupos no incluidos allí, fueron complementados por los asignados en el Índice Biolótico Andino (ABI) (Ríos-Touma et al. 2014). Se calculó el índice BMWP-Col para cada período hidrológico y se promedió su valor por estación. Se consideró el valor del percentil 25 de las estaciones de referencia, como una aproximación adecuada al límite, entre las categorías de la calidad biológica del agua (muy bueno, bueno, regular y malo) y los cortes se realizaron de forma proporcional a la relación exponencial, definiéndose dichos límites, con el 61, 36 y 15\% del percentil 25, de las estaciones de referencia (Tabla 2), de acuerdo con Acosta et al. (2009). Finalmente, se calculó el índice de calidad ecológica de los ríos Andinos ECOSTRIAND, modificado a partir del propuesto por Acosta et al. (2009), que valora de forma global la calidad del ecosistema fluvial, incluyendo la ribera, además de la calidad biológica de las aguas y de la comunidad de macroinvertebrados, usando los valores de los índices BMWP-Col y QBR-And (Tabla 2).

Tabla 2. Propuesta de establecimiento del estado ecológico de las estaciones de muestreo en función de los resultados del BMWP/Col y el QBR-And (índice ECOSTRIAND).

\begin{tabular}{|l|c|c|c|}
\hline \multirow{2}{*}{ BMWP/Col } & \multicolumn{3}{|c|}{ QBR-And } \\
\cline { 2 - 4 } & $\geq 75$ & $\mathbf{4 5 - 7 4}$ & Regular \\
\hline$>102$ & Muy bueno & Bueno & Malo \\
\hline $62-102$ & Bueno & Regular & Pésimo \\
\hline $37-61$ & Regular & Malo & Pésimo \\
\hline$<37$ & Malo & Pésimo & \\
\hline
\end{tabular}


Para contrastar diferencias entre los valores medios de los índices evaluados entre las estaciones de referencia y las alteradas, se utilizó la prueba no paramétrica de Kruskall-Wallis y las comparaciones pareadas con la prueba Mann-Whitney, con el programa estadístico PAST (Hammer et al. 2001).

\section{RESULTADOS Y DISCUSIÓN}

Características del hábitat: De las 12 estaciones evaluadas, se consideraron siete como de referencia $(58,3 \%)$, ubicadas en las quebradas Las Mercedes (E4), Peñoles (E7, E8 y E9) y El Cardal (E10, E11 y E12), las cuales, presentaron vegetación de ribera sin alteraciones o con bajo grado de perturbación antrópica (Tabla 1). Estas estaciones presentaron una temperatura superficial media del agua de $19,8^{\circ} \mathrm{C}$; oxígeno disuelto de $10,0 \mathrm{mg} / \mathrm{L}$; conductividad de $26,2 \mu \mathrm{S} / \mathrm{cm}$ y $\mathrm{pH}$ de 7,8 (Tabla 3). Asimismo, se establecieron cinco estaciones consideradas como alteradas, ubicadas en las quebradas La Magdalena (E1, E2 y E3) y Las Mercedes (E5 y E6), con alta perturbación antrópica, sin cobertura arbórea en sus márgenes, con descargas de aguas residuales, contaminación con insecticidas y fertilizantes, usados en actividades agrícolas y ganaderas, lavado de cabuya, entre otros (Tabla 1). La temperatura superficial del agua presentó un promedio de $18,7^{\circ} \mathrm{C}$; oxígeno disuelto de 9,2mg/L; conductividad de $24,3 \mu \mathrm{S} / \mathrm{cm}$ y $\mathrm{pH}$ de 8,3 (Tabla 3 ); en este sentido, las estaciones alteradas presentaron menor temperatura y mayores valores de $\mathrm{pH}$. La diferencia de temperatura está asociada con la altura sobre el nivel del mar, ya que las estaciones alteradas están ubicadas en zonas de mayor nivel altitudinal, mientras que los mayores valores de $\mathrm{pH}$ pueden estár asociados con la descarga de aguas residuales, insecticidas y fertilizantes, usados en actividades agrícolas y ganaderas.

IHF: Las estaciones de referencia presentaron valores entre 97 y 70, con una media de 79,7 y un coeficiente de variabilidad (C.V.) de 9,8\%; estos valores superiores a 70 puntos indican que el hábitat fluvial es apto para albergar diversos ensamblajes de macroinvertebrados acuáticos, peces y otros grupos de biota acuática. Por su parte, las estaciones alteradas presentaron valores entre 61 y 37, con una media de 50,6 y un C.V. de 17,2\% (Tabla 3), indicando una disminución en la calidad del hábitat para estas últimas. En particular, la estación E1 presentó un valor IHF de 37, lo que indica una menor calidad de hábitat, con baja heterogeneidad, para el desarrollo de comunidades acuáticas. Valores del IHF por debajo de 40 indican serias limitaciones de calidad de hábitat, para el desarrollo de una comunidad bentónica diversa, siendo el óptimo superior a 75 (Pardo et al. 2002; Acosta et al. 2009). El valor medio del IHF fue mayor en las estaciones de referencia y significativamente diferente del valor medio de las estaciones alteradas (Mann-Whitney, $P=0,006)$ y se debe a su relación con la disponibilidad de cobertura bos- cosa de las riberas. De acuerdo con Mercer (2013) y Allan (2004), la pérdida de vegetación riparia es una perturbación que puede generar factores erosivos, que conducen a la sedimentación, a través de lixiviación de partículas de la tierra a la corriente, causando reducciones en la calidad de los sustratos, cambios en el tipo y tamaño de las partículas y la disponibilidad de nutrientes, que afectan la calidad de hábitat.

QBR-And: Las estaciones de referencia presentaron valores entre 95 y 70 de este índice, con una media de 82,9 y un C.V. de $10,6 \%$, mientras que las estaciones alteradas presentaron valores entre 25 y 5 , con una media de 15,0 y un C.V. de 55,8\% (Tabla 3). El percentil 25 de los valores de las 7 estaciones de referencia fue de 75 y solamente E8 presentó un valor inferior. El valor medio del QBR-And fue significativamente mayor en las estaciones de referencia (Mann-Whitney, $\mathrm{P}=0,005)$ y presentó valores altos, de acuerdo a los rangos de calidad de conservación de la vegetación de ribera, propuestos para este índice y se clasificaron como áreas con vegetación ligeramente perturbada y calidad buena. De acuerdo con Wallin et al. (2003), las condiciones de referencia se deben asignar según los diferentes tipos de cuerpos de agua y no necesariamente representan condiciones prístinas y totalmente inalteradas, sino que cierta presión humana se puede presentar, mientras no existan efectos ecológicos o sean mínimos.

En las estaciones de referencia, se observó abundante vegetación nativa en las márgenes y una menor intervención antrópica, aumentando, significativamente, los valores en el índice QBR-And, en relación a las estaciones alteradas, donde se presentaron valores menores, clasificándose dentro del rango de calidad de conservación de la vegetación de ribera, como áreas con degradación extrema y calidad pésima, debido a la ausencia o reducción casi total de la vegetación arbórea de ribera, ocasionada por actividades agrícolas y ganaderas. Según Gergel et al. (2002) y Palma et al. (2009), la vegetación de las márgenes actúa como zonas de amortiguamiento, que detienen los nutrientes, los sedimentos y los demás contaminantes que puedan llegar al cuerpo de agua, desde los suelos adyacentes y las franjas riparias, más anchas, poseen mayor capacidad para disminuir la concentración de herbicidas y el exceso de fertilizantes, a través de la infiltración radicular. En este sentido, la calidad ecológica de los cuerpos de agua está influenciada por los bosques de ribera y los usos del suelo, en actividades antropogénicas (Bojsen \& Barriga, 2002; Novotny et al. 2005), afectando los cambios en las variables físico químicas y la composición del sustrato (Tejerina-Garro et al. 2005).

BMWP/Col: Las estaciones de referencia presentaron valores entre 119 y 98, con una media de 107,4 y un C.V. de 6,4\%, 


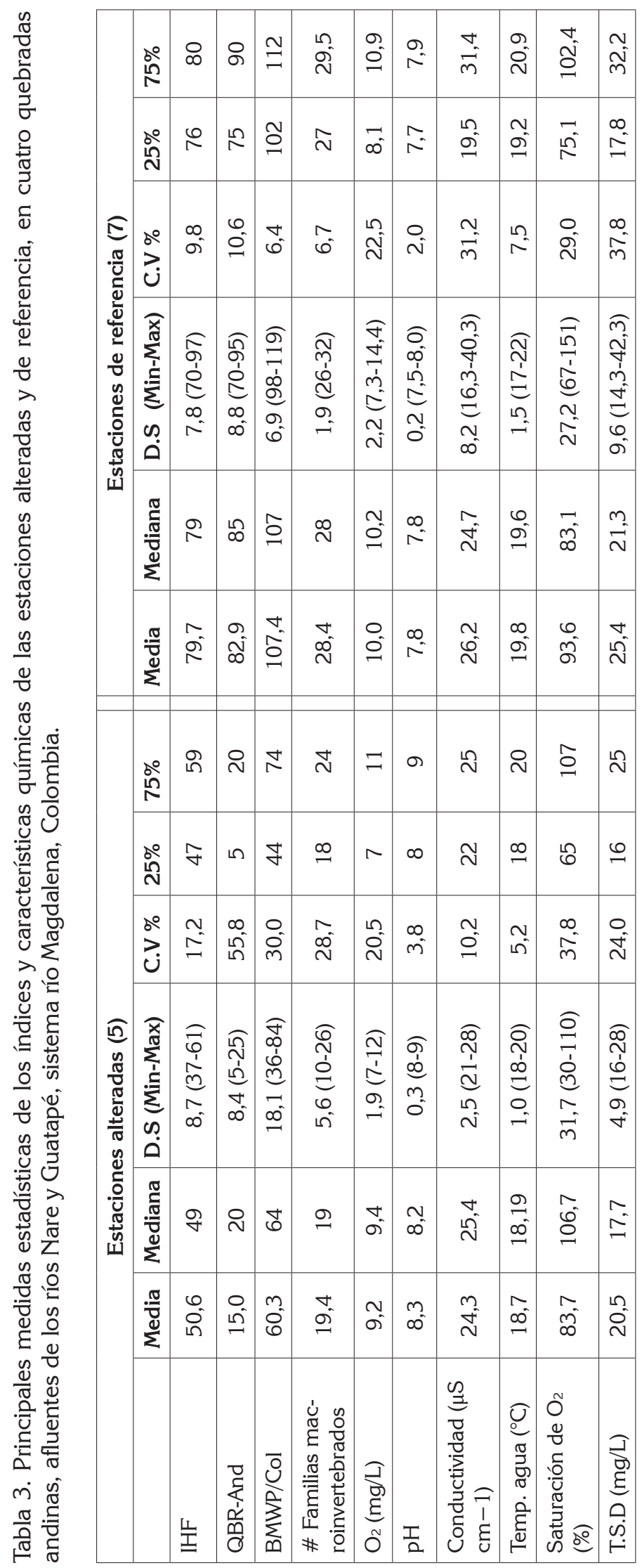


mientras que las estaciones alteradas presentaron valores entre 84 y 36, con una media de 60,3 y un C.V. de 30,0\% (Tabla 3). De acuerdo con este índice, cinco de las estaciones de referencia presentaron valores superiores al valor del percentil 25 de este índice (102), reflejando un estado ecológico muy bueno, con aguas muy limpias, no contaminadas o poco alteradas (E4, E7, E9, E11 y E12) y dos presentaron un estado bueno, con aguas ligeramente contaminadas (E8 y E10). Con relación a las estaciones alteradas, tres de ellas presentaron estado ecológico bueno (E2, E5 y E6), mientras que E1 mostró estado regular, con aguas moderadamente contaminadas y E3 estado malo, con aguas muy contaminadas, debido, posiblemente, al desarrollo de actividades, como lavado de cabuya, que algunos habitantes de la zona realizan en la quebrada La Magdalena y a la posible lixiviación de residuos de insecticidas y de fertilizantes, usados en las actividades agrícolas, realizadas en sus márgenes. El valor medio del BMWP/Col fue significativamente mayor en las estaciones de referencia que el de las estaciones alteradas (Mann-Whitney, $\mathrm{P}=0$,006).
ECOSTRIAND: De acuerdo con este índice, las estaciones de referencia presentaron un estado ecológico muy bueno (E4, E7, E9, E11 y E12) o bueno (E10) y solo una de ellas fue calificada con estado ecológico regular (E8) (Tabla 4), debido, posiblemente, a la disminución de la vegetación riparia y la apertura de algunos claros en el bosque, con presencia de pastos, para ganadería, lo cual, coincide con los resultados de Acosta et al. (2009), donde estaciones que fueron calificadas originalmente como de referencia, resultaron como "malas", según el ECOSTRIAND, debido, especialmente, al deterioro de la vegetación de sus riberas. Por su parte, las estaciones alteradas presentaron un estado ecológico malo (E2, E5 y E6) y pésimo (E1 y E3) (Tabla 4), debido a que muestran contaminación, por las actividades agrícolas de la zona.

Número de familias de macroinvertebrados acuáticos: El número total de familias fue 57 (Tabla 5). Las estaciones de referencia presentaron entre 32 y 26 familias, con una media de 28,4 y un C.V. de $6,7 \%$, mientras que las alteradas pre-

Tabla 4. Valores de los índices aplicados para las diferentes estaciones de muestreo, en cuatro quebradas Andinas, afluentes de los ríos Nare y Guatapé, sistema río Magdalena, Colombia.

\begin{tabular}{|c|c|c|c|c|c|c|}
\hline Estación & $\begin{array}{l}\text { Puntaje de } \\
\text { referencia }\end{array}$ & IHF & QBR-And & \multicolumn{2}{|c|}{$\underline{B M W P / C o l}$} & ECOSTRIAND \\
\hline 1 & 82 & 37 & 20 & 44 & Regular & Pésimo \\
\hline 2 & 80 & 47 & 5 & 64 & Bueno & Malo \\
\hline 3 & 86 & 59 & 20 & 36 & Malo & Pésimo \\
\hline 4 & $120 *$ & 76 & 95 & 119 & Muy bueno & Muy bueno \\
\hline 5 & 94 & 61 & 25 & 84 & Bueno & Malo \\
\hline 6 & 88 & 49 & 5 & 74 & Bueno & Malo \\
\hline 7 & $112 *$ & 79 & 85 & 114 & Muy bueno & Muy bueno \\
\hline 8 & $108^{*}$ & 79 & 70 & 98 & Bueno & Regular \\
\hline 9 & $104^{*}$ & 81 & 75 & 107 & Muy bueno & Muy bueno \\
\hline 10 & $114^{*}$ & 70 & 90 & 100 & Bueno & Bueno \\
\hline 11 & $116^{*}$ & 97 & 90 & 109 & Muy bueno & Muy bueno \\
\hline 12 & $110 *$ & 76 & 75 & 105 & Muy bueno & Muy bueno \\
\hline
\end{tabular}

* Estaciones consideradas como puntos de referencia.

sentaron valores entre 26 y 10, con una media de 19,4 y un C.V. de $28,7 \%$. El percentil 25 de los valores de las estaciones de referencia fue de 27 familias (Tabla 3). El número de familias de macroinvertebrados fue significativamente mayor en las estaciones de referencia que en las alteradas (MannWhitney, $P=0,007$ ), lo cual, se puede deber a la presencia de actividades agropecuarias y pérdida de coberturas arbóreas, observadas en los márgenes de las estaciones alteradas, lo que causa mayores niveles de sedimentación, modificación del sustrato y cambios en la estructura y composición de macroinvertebrados, lo que coincide con lo encontrado por Ríos \& Bailey (2006) y Palma et al. (2009).

El mayor número de familias de macroinvertebrados en las estaciones de referencia, se relaciona con los valores altos en el índice de QBR-And, debido a que la hojarasca producida 
Tabla 5. Familias de macroinvertebrados acuáticos en estaciones alteradas y de referencia.

\begin{tabular}{|c|c|c|c|}
\hline Clase/Orden & Superfamilia/ Familia & Alteradas & Referencia \\
\hline \multirow{8}{*}{ Coleoptera } & Dryopidae & $\mathrm{X}$ & $\mathrm{X}$ \\
\hline & Elmidae & $\mathrm{X}$ & $\mathrm{X}$ \\
\hline & Hidrophilidae & & $\mathrm{X}$ \\
\hline & Limnychidae & & $\mathrm{X}$ \\
\hline & Lutrochidae & & $\mathrm{X}$ \\
\hline & Psephenidae & $\mathrm{X}$ & $\mathrm{X}$ \\
\hline & Ptilodactylidae & $\mathrm{X}$ & $\mathrm{X}$ \\
\hline & Scirtidae & $\mathrm{X}$ & $\mathrm{X}$ \\
\hline \multirow{8}{*}{ Diptera } & Blephariceridae & $\mathrm{X}$ & $\mathrm{X}$ \\
\hline & Ceratopogonidae & & $\mathrm{X}$ \\
\hline & Chironomidae & $\mathrm{X}$ & $\mathrm{X}$ \\
\hline & Dolichopodidae & & $\mathrm{X}$ \\
\hline & Muscidae & $\mathrm{X}$ & \\
\hline & Psychodidae & & $\mathrm{X}$ \\
\hline & Simuliidae & $\mathrm{X}$ & $\mathrm{X}$ \\
\hline & Tipulidae & $X$ & $\mathrm{X}$ \\
\hline \multirow{6}{*}{ Ephemeroptera } & Baetidae & $\mathrm{X}$ & $\mathrm{X}$ \\
\hline & Euthyplociidae & & $\mathrm{X}$ \\
\hline & Leptohyphidae & & $\mathrm{X}$ \\
\hline & Leptophlebiidae & $\mathrm{X}$ & $\mathrm{X}$ \\
\hline & Oligoneuriidae & & $\mathrm{X}$ \\
\hline & Tricorythidae & $X$ & $\mathrm{X}$ \\
\hline \multirow{8}{*}{ Hemiptera } & Belostomatidae & $\mathrm{X}$ & $\mathrm{X}$ \\
\hline & Corixidae & & $\mathrm{X}$ \\
\hline & Gerridae & & $\mathrm{X}$ \\
\hline & Hebridae & $\mathrm{X}$ & $\mathrm{X}$ \\
\hline & Naucoridae & $\mathrm{X}$ & $\mathrm{X}$ \\
\hline & Notonectidae & & $\mathrm{X}$ \\
\hline & Saldidae & $\mathrm{X}$ & $\mathrm{X}$ \\
\hline & Veliidae & $\mathrm{X}$ & $\mathrm{X}$ \\
\hline \multirow{2}{*}{ Lepidoptera } & Crambidae & $\mathrm{X}$ & $\mathrm{X}$ \\
\hline & Pyralidae & $\mathrm{X}$ & $\mathrm{X}$ \\
\hline Megaloptera & Corydalidae & $\mathrm{X}$ & $\mathrm{X}$ \\
\hline \multirow{6}{*}{ Odonata } & Aeshnidae & & $\mathrm{X}$ \\
\hline & Calopterygidae & $\mathrm{X}$ & $\mathrm{X}$ \\
\hline & Coenagrionidae & $\mathrm{X}$ & $\mathrm{X}$ \\
\hline & Gomphidae & & $\mathrm{X}$ \\
\hline & Libellulidae & $\mathrm{X}$ & $\mathrm{X}$ \\
\hline & Megapodagrionidae & & $\mathrm{X}$ \\
\hline Plecoptera & Perlidae & $\mathrm{X}$ & $X$ \\
\hline
\end{tabular}


Continuación Tabla 5.

\begin{tabular}{|c|c|c|c|}
\hline \multirow{4}{*}{} & Glossosomatidae & $\mathrm{X}$ & $\mathrm{X}$ \\
\cline { 2 - 4 } & Helicopsychidae & $\mathrm{X}$ & $\mathrm{X}$ \\
\cline { 2 - 4 } & Hydrobiosidae & $\mathrm{X}$ & $\mathrm{X}$ \\
\cline { 2 - 4 } & Hydropsychidae & $\mathrm{X}$ & $\mathrm{X}$ \\
\cline { 2 - 4 } & Hydroptilidae & $\mathrm{X}$ & $\mathrm{X}$ \\
\cline { 2 - 4 } Trichoptera & Leptoceridae & $\mathrm{X}$ & $\mathrm{X}$ \\
\hline \multirow{3}{*}{$*$ Hydracarina } & Odontoceridae & $\mathrm{X}$ & $\mathrm{X}$ \\
\cline { 2 - 4 } & Philopotamidae & $\mathrm{X}$ & $\mathrm{X}$ \\
\hline \multirow{3}{*}{ Gastropoda } & Eylaoidea & $\mathrm{X}$ & $\mathrm{X}$ \\
\hline & Hygrobatoidea & $\mathrm{X}$ \\
\hline Gordioidea & Basommatophora & $\mathrm{X}$ & $\mathrm{X}$ \\
\hline Clitellata & Ampullariidae & $\mathrm{X}$ & \\
\hline Tricladida & Planorbidae & $\mathrm{X}$ & $\mathrm{X}$ \\
\hline *Turbellaria & *Gordiidae & $\mathrm{X}$ & $\mathrm{X}$ \\
\hline
\end{tabular}

*Uso de puntajes asignados en el Índice Biolótico Andino (ABI) (Ríos-Touma et al. 2014).

por el dosel genera una mayor heterogeneidad de hábitats, disponibles para las comunidades biológicas. Al respecto, se conoce que los patrones de diversidad se relacionan con el bosque de ribera, porque la hojarasca producida por el dosel genera una mayor heterogeneidad de hábitats y recursos disponibles para las comunidades biológicas (Teresa \& Casatti, 2010).

En síntesis, los altos valores de IHF, en estaciones de referencia, coincidieron con valores significativamente mayores de los índices QBR-And, BMWP/col y ECOSTRIAND, con relación a las estaciones alteradas. Al respecto, Pardo et al. (2002) mencionan que es evidente la importancia de la alteración del hábitat sobre las comunidades de invertebrados acuáticos, ya que la disminución de los valores del IHF y el número de familias de invertebrados se corresponden con un gradiente de disminución de la calidad biológica. Los cinco sitios considerados alterados presentaron alta transformación antrópica, vegetación ribereña reducida o ausente en los dos márgenes, predominio de pastos y actividades agrícolas y bajos valores en los índices antes mencionados, lo que indica una menor calidad ecológica. A pesar de que el índice BMWP/Col usado para evaluar la calidad hídrica presenta tres de las cinco estaciones alteradas con calidad hídrica buena, los índices QBR-And, IHF, ECOSTRIAND y el número de familias de macroinvertebrados muestran que existe una disminución significativa de la calidad ecológica en las estaciones alteradas, con relación a las de referencia. Finalmente, el uso de índices físicos y biológicos se ve como una herramienta útil para la determinación de la calidad ecológica de los ecosistemas acuáticos andinos, teniendo en cuenta que la heterogeneidad espacial y temporal de los hábitats fluviales permite la presencia de mayor diversidad. Su utilización puede ayudar a establecer acciones de protección de las quebradas y hábitats ribereños e identificar tramos de ríos con escasa o nula intervención humana y en muy buen estado ecológico, que puedan ser sugeridos para la creación o declaración de áreas protegidas, como reservas naturales fluviales, que garanticen el mantenimiento de su calidad ecológica. Asimismo, el uso de los ensamblajes de macroinvertebrados acuáticos es una herramienta útil para determinar la calidad ecológica de los cuerpos hídricos, debido a las respuestas diferenciadas de los diversos grupos taxonómicos a la degradación por influencia humana; no obstante, aún es necesario ampliar la información sobre los rangos de tolerancia de los organismos a las diferentes presiones antrópicas y las respuestas específicas de las diferentes comunidades bióticas.

Para el área de estudio, las siete estaciones establecidas como de referencia cumplieron con las condiciones de conservación biológica y física del hábitat, que permiten valorarlas como tal, de acuerdo a los resultados de los índices IHF, QBR-And y ECOSTRIDAND y a que no presentaron presencia de basuras, de contaminación orgánica evidente o derivación del caudal; sin embargo, se evidenció un gradiente de calidad, en el que las estaciones E4, E7, E9, E11 y E12 presentaron los valores más altos de IHF y QBR-And, 
así como calidad muy buena para los índices BMWP/col y ECOSTRIAND, seguidas de la estación E10, que presentó calidad buena, para los índices BMWP/col y ECOSTRIAND y un menor valor de IHF y de la estación E8, que presentó calidad buena, para el índice BMWP/col y regular para el índice ECOSTRIAND, con un menor valor de QBR-And, debido a que presentó algunos tramos con presencia de pastos y disminución de la vegetación riparia.

La aplicación de los distintos índices en relación a su sensibilidad para diagnosticar los cambios en el uso del suelo, mostraron que el índice ECOSTRIAND presentó un mejor poder discriminatorio entre estaciones de muestreo, ya que combina los valores de los índices BMWP-Col y QBR-And y valora, de forma global, la calidad del ecosistema fluvial, incluyendo la ribera, además de la calidad biológica de las aguas y de la comunidad de macroinvertebrados.

Conflictos de intereses: El manuscrito fue preparado y revisado con la participación de todos los autores, quienes declaramos que no existe conflicto de intereses que ponga en riesgo la validez de los resultados presentados. Financiación: Información obtenida en el proyecto "Estudio de la biología, ecología y diversidad genética de las poblaciones naturales de sabaleta Brycon henni en las cuencas de los ríos Nare y Guatapé, Antioquia", financiado por la empresa ISAGEN S.A. E.S.P.; la Corporación Autónoma Regional de los Ríos Negro y Nare -CORNARE y la Universidad Nacional de Colombia.

\section{BIBLIOGRAFÍA}

1. ACOSTA, R.; RÍOS, B.; RIERADEVALL, M.; PRAT, N. 2009. Propuesta de un protocolo de evaluación de la calidad ecológica de ríos Andinos (C.E.R.A) y su aplicación en dos cuencas en Ecuador y Perú. Limnetica. 28(1):35-64.

2. ALLAN, J.D. 2004. Landscapes and riverscapes: the influence of land use on stream ecosystems. Ann. Rev. Ecol. Evol. Syst. 35:257-284.

3. ANDERSON, E.P.; MALDONADO-OCAMPO, J. 2011. A regional perspective on the diversity and conservation of tropical Andean. Conserv. Biol. 25(1):30-39.

4. BAILEY, R.; NORRIS, R.; REYNOLDSON, T. 2004. Bioassessment of Freshwater Ecosystems using the Reference Condition Approach. Kluwer Academic Publishers, Boston. 170p.

5. BARBOUR, M.; SWIETLIK, W.; JACKSON, S.; COURTEMANCH, D.; DAVIES, S.; YODER, C. 2000. Measuring the attainment of biological integrity in the USA: a critical element of ecological integrity. Hydrobiologia. 422/423:453-464.

6. BOJSEN, B.H.; BARRIGA, R. 2002. Effects of deforestation on fish community structure in Ecuadorian Amazon streams. Freshwater Biol. 47:2246-2260.

7. BONADA, N.; PRAT, N.; MUNNÉ, A.; RIERADEVALL, M.; ALBA-TERCEDOR, J.; ÁLVAREZ, M.; AVILÉS, J.; CASAS, J.; JÁIMEZ-CUÉLLAR, P.; MELLADO, A.; ORTEGA, M.; PARDO, I.; ROBLES, S.; SAÍNZ-CANTERO, C.; SÁNCHEZ-ORTEGA, A.; SUÁREZ, M.L.; TORO, M.; VIDAL-ABARCA, M.R.; VIVAS, S.; ZAMORA-MUÑOZ, C. 2002. Criterios para la selección de condiciones de referencia en los ríos mediterráneos. Resultados del proyecto GUADALMED1. Limnetica, 21(3-4):99-114.

8. COMPIN, A.; CÉRÉGHINO, R. 2003. Sensitivity of aquatic insect species richness to disturbance in the Adour-Garonne stream system (France). Ecol. Indic. 3(2):135-142.

9. GERGEL, S.E.; TURNER, M.G.; MILLER, J.R.; MELACK, J.M.; STANLEY, E.H. 2002. Landscape indicators of human impacts to riverine systems. Aquat Sci. 64:118-128.

10. GIRALDO, L.P.; CHARÁ, J.; ZÚĨIIGA, M.C.; CHARÁSERNA, A.; PEDRAZA, G. 2014. Impacto del uso del suelo agropecuario sobre macroinvertebrados acuáticos en pequeñas quebradas de la cuenca del río La Vieja (Valle del Cauca, Colombia). Rev. Biol. Trop. 62(2):203-219.

11. HAMMER, Ø.; HARPER, D.; RYAN. P. 2001. PAST: Paleontological statistics software package for education and data analysis. Palaeontología Electrónica [Computer program]. Retrived from http://palaeoelectronica. org/2001_1/past/issue1_01.htm.

12. HERMAN, M.R.; NEJADHASHEMI, A.P. 2015. A review of macroinvertebrate-and fish-based stream health indices. Ecohydrol. Hydrobiol.15(2):53-67.

13. HUGHES, R. 1995. Defining acceptable biological status by comparing with reference conditions. In Davis, W.S.; SIMON, T.P. (Eds.). Biological assessment and criteria: tools for water resource planning and decision making. Boca Raton: CRC Press Inc. 432p.

14. JACOBSEN, D. 1998. The effect of organic pollution on the macroinvertebrate fauna of Ecuadorian highland streams. Archiv für Hydrobiologie 143(2):179-195. 
15. KARR, J. 1991. Biological integrity: a long-neglected aspect of water resource management. Ecol. Appl. $1: 66-84$.

16. KARR, J. 1981. Assessment of biotic integrity using fish communities. Fisheries. 6:21-27.

17. KERANS, B.L.; KARR, J.R. 1994. A benthic index of biological integrity (B-IBI) for rivers of the Tennessee valley. Ecol. Appl. 4:768-785.

18. LIGEIRO, R.; HUGHES, R.; KAUIFMANN, P.; MACEDO, D.; FIRMIANO, K.; FERREIRA, W.; OLIVEIRA, D.; MELO, A.; CALLISTO, M. 2013. Defining quantitative stream disturbance gradients and the additive role of habitat variation to explain macroinvertebrate taxa richness. Ecol. Ind. 25:45-57.

19. MANCERA-RODRÍGUEZ, N.J.; CASTELLANOS-BARLIZA, J.; URREGO-BALLESTAS, D. 2016. Biología reproductiva de Saccodon dariensis (Teleostei: Parodontidae) en afluentes del río Guatapé, cuenca del río Magdalena, Colombia. Rev. Biol. Trop. 64(2):635653.

20. MERCER, E.; MERCER, T.; SAYOK, A. 2013. Effects of forest conversions to oil palm plantations on freshwater macroinvertebrates: a case study from Sarawak, Malaysia. J. Land Use Sci. 9(3):260-277.

21. MORENO, P.; FRANÇA, J.; FERREIRA, W.; PAZ, A.; MONTEIRO, I.; CALLISTO, M. 2009. Use of the BEAST model for biomonitoring water quality in a neotropical basin. Hydrobiologia. 630:231-242.

22. MUNNÉ, A.; SOLÀ, C.; PAGÈS, J. 2006. HIDRI: Protocolo para la valoración de la calidad hidromorfológica de los ríos. Barcelona, España: Agència Catalana de l'Aigua. 160p.

23. MUNNÉ, A.; PRANT, N.; SOLÀ, C.; BONADA, N.; RIERADEVALL, M. 2003. A simple field method for assessing the ecological quality of riparian habitat in rivers and streams. QBR index. Aquat Conserv. 13:147164.

24. NOVOTNY, V.; BARTOSOVA, A.; O'REILLY, N.; EHLINGER, T. 2005. Unlocking the relationship of biotic integrity of impaired waters to anthropogenic stresses. Water Res. 39(1):184-198.

25. PALMA, A.; FIGUEROA, R.; RUIZ, V. 2009. Evaluación de ribera y hábitat fluvial a través de los índices QBR e IHF. Gayana 73(1):57-63.
26. PARDO, I.; ÁLVAREZ, M.; CASAS, J.; MORENO, J.L.; VIVAS, S.; BONADA, N.; ALBA-TERCEDOR, J.; JAIME-CUELLAR, P.; MOYA, G.; PRANT, N.; ROBLES, S.; SUAREZ, L.M.; TORO, M.; VIDAL- ABARCA, R.M. 2002. El hábitat de los ríos mediterráneos diseño de un índice de diversidad de hábitat. Lim. 21(3-4):115134.

27. PILIÈREA, A.; SCHIPPERA, A.; BREUREA, A.; POSTHUMAB, L.; ZWARTB, D.; DYERC, S.; HUIJBREGTS, M. 2014. Comparing responses of freshwater fish and invertebrate community integrity along multiple environmental gradients. ECOL INDIC. 43:215-226.

28. PIÑÓN-FLORES, M.A.; PÉREZ-MUNGUÍA, R.M.; TORRES-GARCÍA, U.; PINEDA-LÓPEZ, R. 2014. Integridad biótica de la microcuenca del río Chiquito, Morelia Michoacán, México, basada en el ensamblaje de macroinvertebrados acuáticos. Rev Biol Trop. 62(2):219-229.

29. REYNOLDSON, T.; NORRIS, R.; RESH, V.; DAY, K.; ROSENBERG, D. 1997. The reference condition: A comparison of multimetric and multivariate approaches to assess water-quality impairment using benthic macroinvertebrate. Nor. Amer. Bent. Soc. 16(4):833-852.

30. RÍOS, S.L.; BAILEY, R.C. 2006. Relationship between riparian vegetation and stream benthic communities at three spatial scales. Hydrobiologia. 553:153-160.

31. RÍOS-TOUMA, B.; ACOSTA, R.; PRAT, N. 2014. The Andean Biotic Index (ABI): revised tolerance to pollution values for macroinvertebrate families and index performance evaluation. Rev. Biol. Trop. 62(2):249-273.

32. ROLDÁN, G.; RAMÍREZ, J. 2008. Fundamentos de limnología neotropical. Medellín, Colombia: Universidad de Antioquia. 440p.

33. ROLDÁN, G. 2003. Bioindicación de la calidad del agua en Colombia: Uso del método BMWP/Col. Medellín, Colombia: Universidad de Antioquia. 175p.

34. STODDARD, J.; LARSEN, D.; HAWKINS, C.; JOHNSON, R.; NORRIS, R. 2006. Setting expectations for the ecological condition of running waters: the concept of reference condition. Ecol. Appl. 16:1267-1276.

35. SUÁREZ-ALONSO, M.L.; VIDAL-ABARCA, M.R.; SÁNCHEZ-MONTOYA, M.M.; ALBA-TERCEDOR, J.; ÁLVAREZ, M.; AVILÉS, J.; BONADA, N.; CASAS, J.; JÁIMEZ-CUÉLLAR, P.; MUNNÉ, A.; PARDO, I.; PRAT, 
N.; RIERADEVALL, M.; SALINAS, J.M.; TORO, M.; VIVAS, S. 2002. La ribera de los ríos mediterráneos y su calidad: el uso del índice QBR. Limnetica 21(34):135-148.

36. TEJERINA-GARRO, F.; MALDONADO, M.; IBÁÑEZ, C.; PONT, D.; ROSET, N.; OBERDORFF, T. 2005. Effects of natural and anthropogenic environmental changes on riverine fish assemblages: a framework for ecological assessment of rivers. Braz. Arch. Biol. Technol. 48(1):91-108.

37. TERESA, F.; CASATTI, L. 2010. Importância da vegetação ripária em região intensamente desmatada no sudeste do Brasil: um estudo com peixes de riacho. J Aquat Sci. 5(3):444-453.
38. WALLIN, M.; WIEDERHOLM, T.; JOHNSON, R.K. 2003. Guidance on establishing reference conditions and ecological status class boundaries for inland surface waters. Sweden: CIS Working Group 2.3. REFCOND. 93p.

39. WHITTIER, T.; STODDARD, J.; LARSEN, D.; HERLIHY, A. 2007. Selecting reference sites for stream biological assessments: best professional judgment or objective criteria. J. N. Am. Benthol. Soc. 26(2):349360.

Recibido: Mayo 18 de 2017

Aceptado: Noviembre 20 de 2017

Cómo citar:

Galeano-Rendón, E.; Monsalve-Cortes, L.M.; Mancera-Rodríguez, N.J. 2017. Evaluación de la calidad ecológica de quebradas andinas en la cuenca del río Magdalena, Colombia. Rev. U.D.C.A Act. \& Div. Cient. 20(2): 413-424. 